\title{
Treatment with a Ginkgo biloba extract, EGb 76I, inhibits excitotoxicity in an animal model of spinocerebellar ataxia type 17
}

This article was published in the following Dove Press journal:

Drug Design, Development and Therapy

18 February 2016

Number of times this article has been viewed

\author{
Ding-Siang Huangl,* \\ Hsuan-Yuan Lin ${ }^{1,2, *}$ \\ Guey-Jen Lee-Chen' \\ Hsiu-Mei Hsieh-Li' \\ Chung-Hsin $\mathrm{Wu}^{\prime}$ \\ Jung-Yaw Lin ${ }^{1,2}$
}

'Department of Life Science, National Taiwan Normal University, ${ }^{2}$ Institute of Biochemistry and Molecular Biology, National Taiwan University, Taipei City, Taiwan, Republic of China

*These authors contributed equally to this work
Correspondence: Jung-Yaw Lin Translational Medicine Laboratory, Department of Life Science, No 88, Section 4, Tingzhou Road, Wenshan District, Taipei City II6, Taiwan, Republic of China

Tel +8862 77346269

Email linjy@ntu.edu.tw

Chung-Hsin Wu

Neurophysiology and Neuroethology Laboratory, Department of Life Science, No 88, Section 4, Tingzhou Road,

Wenshan District, Taipei City II6,

Taiwan, Republic of China

Tel +8862 77346364

Email megawu@ntnu.edu.tw
Abstract: Spinocerebellar ataxia type 17 (SCA 17) is a polyglutamine disease caused by the expansion of CAG/CAA repeats in the TATA box-binding protein (TBP) gene. The Ginkgo biloba extract, EGb 761, contains flavonoids and terpenoids with a potential use for the treatment of neurodegenerative diseases such as Alzheimer's and Parkinson's diseases. The neuroprotective effects of EGb 761 are obvious, but whether the EGb 761 has therapeutic effects in SCA 17 is still unclear. To manage our issues, we have generated TBP/79Q-expressing SH-SY5Y cells and SCA 17 transgenic mice with the mutant $h T B P$ gene. In in vitro experiment, we observed that the EGb 761 treatment decreased the amount of sodium dodecyl sulfate-insoluble proteins in the TBP/79Q-expressing SH-SY5Y cells. We further found that the EGb 761 treatment could inhibit excitotoxicity and calcium influx and reduce the expression of apoptotic markers in glutamate-treated SH-SY5Y neuroblastoma cells. In in vivo experiment, we observed that the EGb 761 treatment ( $100 \mathrm{mg} / \mathrm{kg}$ intraperitoneal injection per day) could relieve the motor deficiencies of the SCA 17 transgenic mice. Our findings provide evidence that the EGb 761 treatment can be a remedy for SCA 17 via suppressing excitotoxicity and apoptosis in SCA 17 cell and animal models. Therefore, we suggest that EGb 761 may be a potential therapeutic agent for treating SCA 17.

Keywords: spinocerebellar ataxia type 17, excitotoxicity, EGb 761, polyQ diseases, apoptosis

\section{Introduction}

Spinocerebellar ataxia type 17 (SCA 17) is a polyglutamine (polyQ) disease caused by the expansion of $\mathrm{CAG} / \mathrm{CAA}$ repeats ( $>43$ repeats) in the TATA box-binding protein (TBP) gene, leading to protein aggregation in patients with SCA $17 .^{1-3}$ There is increasing evidence that excitotoxicity and polyQ diseases are related to each other. The sodium dodecyl sulfate (SDS)-resistant aggregation of insoluble proteins is increased after the glutamate-induced influx of calcium, and the aggregation is reduced by the inhibition of calcium influx in a model of SCA $3 .{ }^{4}$ Moreover, the activation of calciumdependent proteolytic calpains aggravates SCA 3 pathology by increasing protein aggregation; however, calpastatin, an inhibitor of calpains, reduces aggregation. ${ }^{5,6}$ Moreover, the intracellular calcium concentration is elevated in neurons that are sensitive to glutamate-induced cell death in Huntington's disease. ${ }^{7,8}$ These findings suggest that disrupted calcium signaling may lead to protein aggregation and neuronal death in polyQ diseases. Thus, the inhibition of calcium overloading may be therapeutic by preventing the pathological alterations in SCA 17. 
The EGb 761, a standard extract prepared from the leaves of Ginkgo biloba, has pharmacological effects in various neurodegenerative diseases. ${ }^{9}$ Pharmacological studies had shown that the G. biloba extract, EGb 761, is an antioxidant agent that alleviates ischemia, oxidative stress, and $\beta$-amyloid-induced toxicity. The EGb 761 has been used in several neurological diseases such as Alzheimer's disease, Parkinson's disease, and dementia. ${ }^{10-13}$ The neuroprotective effects of the EGb 761 are obvious, but whether the EGb 761 has therapeutic effects in SCA 17 is still unclear.

In the present study, we have generated TBP/79Q-expressing SH-SY5Y cells with inducible green fluorescent protein expression and SCA 17 transgenic mice with the mutant hTBP gene driven by the Purkinje-specific protein (Pcp2/L7) gene promoter. $^{14,15}$ The possible effects of the EGb 761 in pathological alterations of SCA 17 were investigated in glutamate-induced excitotoxicity model, TBP-expressing cells, and SCA 17 transgenic mice as in vivo and in vitro models of SCA 17.

\section{Materials and methods Materials}

EGb 761, containing 24\% Ginkgo flavone glycosides (including quercetin, kaempferol, and isorhamnetin) and 6\% terpenlactones (including ginkgolides A, B, C, and bilobalide), is a standardized extract of G. biloba (Dr Willmar Schwabe Pharmaceuticals, Karlsruhe, Germany). 3-(4,5-Dimethylthiazole-2-yl)-2,5-diphenyltetrazolum bromide (MTT), L-glutamic acid monosodium salt monohydrate, and MK-801 were purchased from Sigma-Aldrich Co. (St Louis, MO, USA). Fluo-4 AM was obtained from Thermo Fisher Scientific (Waltham, MA, USA). Trypsin-ethylenediaminetetraacetic acid (0.5\%) and penicillin/streptomycin were purchased from Thermo Fisher Scientific. Fetal bovine serum (FBS) was obtained from Corning Incorporated (Corning, NY, USA). Antibodies specific for calpain 2, calbindin, cleaved caspase 3 , and cleaved poly(adenosine diphosphate-ribose) polymerase (PARP) were produced by Cell Signaling Technology (Danvers, MA, USA). Antibodies against Bax and actin were purchased from EMD Millipore (Billerica, MA, USA), and antibodies against spectrin, transcription factor II D, and Bcl-2 were obtained from Santa Cruz Biotechnology Inc. (Dallas, TX, USA). Horseradish peroxidase (HRP)-conjugated secondary goat anti-mouse and goat anti-rabbit antibodies were purchased from EMD Millipore.

\section{Cell culture}

Human neuroblastoma SH-SY5Y cells were cultured in a 1:1 mixture of Dulbecco's Modified Eagle's Medium and Ham's Nutrient Mixture F-12 containing 10\% fetal bovine serum, $100 \mathrm{U} / \mathrm{mL}$ penicillin, and $100 \mu \mathrm{g} / \mathrm{mL}$ streptomycin. SH-SY5Y cells were cultured with or without the inducible TBP/36Q and TBP/79Q expression constructs. TBP expression was induced by the addition of $10 \mu \mathrm{g} / \mathrm{mL}$ doxycycline. The culture medium was changed every 48 hours, and cells were grown at $37^{\circ} \mathrm{C}$ in the presence of $5 \% \mathrm{CO}_{2}$. Cell experiments were approved by the Biological Experimental Safety Committee at the National Taiwan Normal University.

\section{Cell viability assay}

A total of 104 SH-SY5Y cells/well in a $100 \mu \mathrm{L}$ culture medium were grown in a 96-well plate for 24 hours to reach $\sim 60 \%$ confluence. Cells were treated with vehicle, EGb $761(5 \mu \mathrm{g} / \mathrm{mL}, 10 \mu \mathrm{g} / \mathrm{mL}$, and $20 \mu \mathrm{g} / \mathrm{mL})$, or $10 \mu \mathrm{M}$ MK-801 ( $N$-methyl-D-aspartate [NMDA] receptor antagonist) for 1 hour, and then they were incubated in the presence of $100 \mathrm{mM}$ glutamate for 24 hours. The number of viable cells was compared between the control and treated conditions. The culture medium was supplemented with MTT $(0.5 \mathrm{mg} / \mathrm{mL})$ for 3 hours, and then $10 \% \mathrm{SDS} / \mathrm{HCl}$ buffer was added to each well. The number of viable cells was determined by the measurement of MTT absorbance at $570 \mathrm{~nm}$ using a microplate reader (BioTek Instruments, Winooski, Vermont, USA).

\section{Measurement of calcium influx}

To measure the calcium concentration in viable cells, Fluo-4 AM, a green fluorescent calcium indicator, was used. SH-SY5Y cells were pretreated with vehicle, $20 \mu \mathrm{g} / \mathrm{mL}$ EGb 761, or $10 \mu \mathrm{M}$ MK-801 for 1 hour, then the cells were washed twice in phosphate-buffered saline (PBS) and stained in the dark with $10 \mu \mathrm{M}$ Fluo-4 AM for 1 hour at $37^{\circ} \mathrm{C}$. Cells were washed twice in PBS and exposed to $100 \mathrm{mM}$ glutamate for 1 hour in Locke's buffer. Cells were then centrifuged at $1,000 \mathrm{rpm}$ and resuspended in PBS, and the fluorescence intensity of cells, representing the cytosolic calcium concentration, was measured using the BD FACSCalibur flow cytometer (BD, Franklin Lakes, NJ, USA).

\section{Western blotting experiment and analysis} SH-SY5Y cells were pretreated with vehicle, $20 \mu \mathrm{g} / \mathrm{mL}$ $\mathrm{EGb} 761$, or $10 \mu \mathrm{M} \mathrm{MK}-801$ for 1 hour, and then they were incubated in the presence of $100 \mathrm{mM}$ glutamate for 24 hours. In TBP-expressing cells (TBP/36Q-enhanced green fluorescent protein [EGFP] or TBP/79Q-EGFP cells), the cells were pretreated with vehicle and $20 \mu \mathrm{g} / \mathrm{mL}$ EGb 761 for 1 hour, and $10 \mu \mathrm{g} / \mathrm{mL}$ doxycycline was added to induce TBP/36Q or TBP/79Q expression for 5 days. Cells were harvested and lysed in radioimmunoprecipitation assay buffer (50 mM Tris- $\mathrm{HCl}, 150 \mathrm{mM} \mathrm{NaCl}, 6 \mathrm{mM}$ 
sodium deoxycholate, 1\% NP-40, pH 7.4) supplemented with protease inhibitors (Hoffman-La Roche Ltd., Basel, Switzerland) on ice for 10 minutes. Cell lysates were sonicated and centrifuged at 13,000 rpm for 15 minutes at $4^{\circ} \mathrm{C}$ to collect the supernatants, and protein concentration was measured using the BCA Protein Assay Kit (Thermo Fisher Scientific). Proteins were separated on $12.5 \%$ or $15 \%$ SDS polyacrylamide gels, and they were transferred to polyvinylidene difluoride membranes. The antibodies used in this study were $\beta$-actin, calpain 2, Bax, Bcl-2, cleaved caspase 3 , cleaved PARP, transcription factor II D, and $\alpha$-spectrin (Cell Signaling Technology). Antibodies were detected by suitable HRP-conjugated secondary antibody, protein-immunoreactive bands were visualized by the enhanced chemiluminescence substrate (EMD Millipore), and the band intensities were quantified with the Image J analysis software (Version 1.48t; Wayne Rasnabd, Washington, DC, USA).

\section{Animals}

SCA 17 transgenic mice in the experiment were obtained from the laboratory of Professor HM Hsieh-Li (National Taiwan Normal University, Taipei City, Taiwan, Republic of China). ${ }^{15}$ They were generated with the mutant hTBP gene driven by the Purkinje-specific protein (Pcp2/L7) gene promoter. The mice with the expanded hTBP allele developed ataxia within 2-5 months. They were identified using polymerase chain reaction with a forward primer from the Pcp2/L7 promoter (L7-F: 5'-TATGGTGAGAGCAGAGATGG-3') and a backward primer from hTBP cDNA (hTBP-3R: 5'-CTGCTGGGACGTTGACTGCTG-3'). SCA 17 transgenic mice and their wild-type (FVB/N mice) were housed in a temperature-controlled environment at $22^{\circ} \mathrm{C} \pm 2{ }^{\circ} \mathrm{C}$ with a 12-hour light/dark cycle. All mice were housed and maintained on a 12:12-hour light-dark cycle with water and food ad libitum. Two groups of three transgenic mice each and littermates received water vehicle or $100 \mathrm{mg} / \mathrm{kg}$ EGb 761 intraperitoneally every other day from the age of 4 weeks to 24 weeks. Animal experiments were approved by the Institutional Animal Care and Use Committee at the National Taiwan Normal University.

\section{Rotarod performance}

Rotarod performance was used to examine the motor coordination of SCA 17 transgenic mice. The mice were tested on a rotarod apparatus (Ugo Basile, Varese, Italy) starting at $5 \mathrm{rpm}$ and accelerating to $15 \mathrm{rpm}$ over a period of 2 minutes and then maintaining a constant speed of $15 \mathrm{rpm}$ for 2 minutes. The mice were rested for 30 minutes between trials and trained at $5 \mathrm{rpm}$ for 10 minutes/day for 3 days at 8 weeks of age to become acquainted with the rotarod apparatus. Then, they were tested three times a week from the age of 9 weeks to 24 weeks, and the latency period was recorded before the mice fell off the rotating rod.

\section{Immunohistochemistry}

Wild-type and SCA 17 transgenic mice were cardiac perfused first with PBS and then fixed with $4 \%$ formaldehyde (EM grade) at the age of 24 weeks. Cerebellar specimens were then embedded in paraffin, cut into sections $(5 \mu \mathrm{m})$, and placed on adhesion microscope slides (Thermo Fisher Scientific). By using the heat-induced epitope retrieval method, the sections were separately stained at room temperature for 1 hour with antibodies of calbindin (1:500; Cell Signaling Technology) or TBP $(1: 1,000$; Santa Cruz Biotechnology Inc.). Detection was performed by incubation with biotinylated secondary antibodies (Novolink ${ }^{\mathrm{TM}}$ Polymer Detection System 1; Leica Microsystems, Wetzlar, Germany), for 30 minutes at room temperature, followed by 30 -minute incubation with avidin-biotin-HRP complex (Novolink ${ }^{\mathrm{TM}}$ Polymer Detection System 1). Visualization was performed with 3,3'-diaminobenzidine Chromogen (Novolink ${ }^{\mathrm{TM}}$ Polymer Detection System 1) and counterstained with hematoxylin (Novolink ${ }^{\mathrm{TM}}$ Polymer Detection System 1) following the supplier's protocol.

\section{Statistical analysis}

Statistical analyses were performed with Student's $t$-test or one-way analysis of variance test (using Bonferroni's test for post hoc comparisons) where appropriate, and the results are shown as mean \pm standard error of mean values. Results were considered significant at $P<0.05$.

\section{Results}

The effect of EGb 76I on glutamateinduced cytotoxicity

Glutamate is the major excitatory neurotransmitter in the central nervous system, but high levels of released glutamate can cause neuronal dysfunction via the activation of NMDA receptors, and then inducing excitotoxicity in the cells. ${ }^{16,17}$ To examine whether EGb 761 alleviates excitotoxicity, SHSY5Y cells were pretreated with various concentrations of EGb $761(0-20 \mu \mathrm{g} / \mathrm{mL})$ for 1 hour followed by an incubation with glutamate $(100 \mathrm{mM})$ for 24 hours, and finally the cell viability was measured using the MTT assay. Cell survival was significantly increased in the presence of $20 \mu \mathrm{g} / \mathrm{mL} \mathrm{EGb}$ 761 or the NMDA receptor antagonist MK-801 (10 $\mu \mathrm{M}$; Figure 1A). 
A

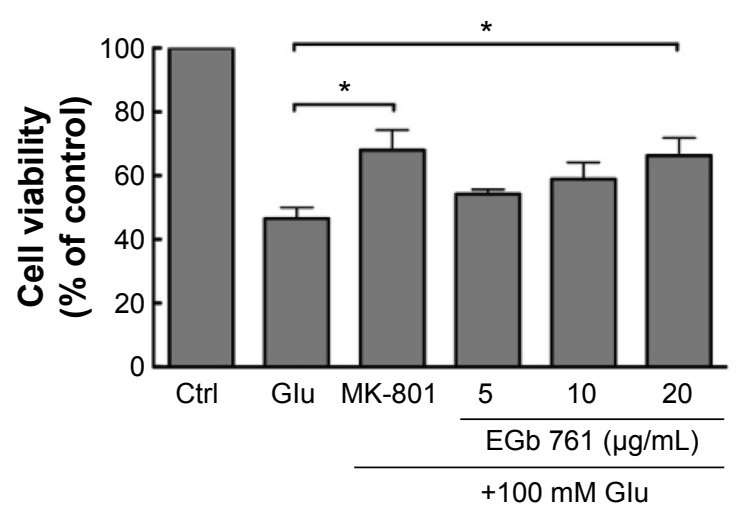

B

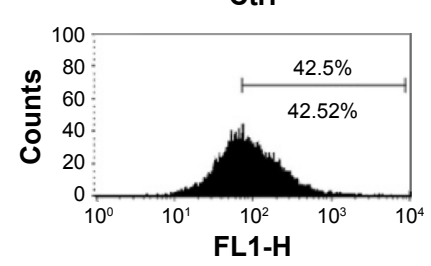

Glu + MK-801

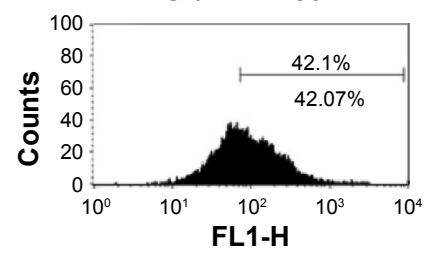

Glu

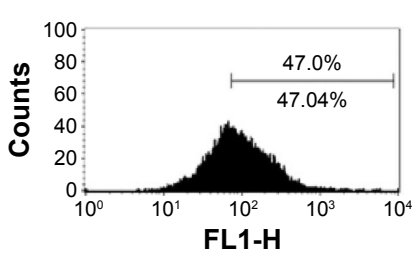

Glu + EGb 761

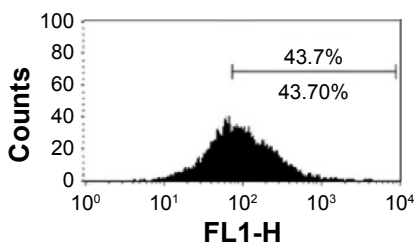

C
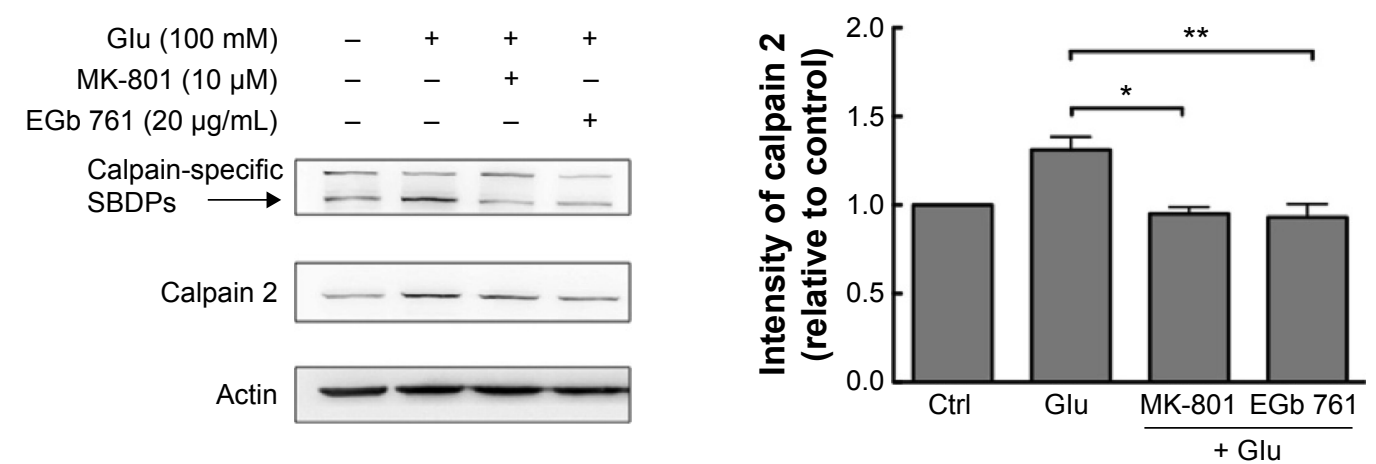

Figure I Protective effects of EGb 76I in glutamate-induced excitotoxicity of SH-SY5Y cells.

Notes: (A) Viability of SH-SY5Y neuroblastoma cells is measured after 24 hours of glutamate treatment (Glu, $100 \mathrm{mM})$. EGb $76 \mathrm{I}(5-20 \mu \mathrm{g} / \mathrm{mL})$ is added in the presence of glutamate. MK-80I (I0 $\mu \mathrm{M})$ is an NMDA receptor antagonist, which is used as positive control for the glutamate-induced cell death. (B) Representative images of Fluo-4 AM (FLI) labeling of SH-SY5Y cells analyzed by flow cytometry. (C) Cells are harvested after glutamate treatment for 24 hours and immunoblotted with the anti-calpain 2 and anti- $\alpha$-spectrin antibodies (left panel). Expression of calpain 2 is normalized for the internal control actin, and data (mean \pm SEM, $n=3$ ) are presented as the relative intensity of calpain 2 (right panel). $* P<0.05 ; * * P<0.0$ I.

Abbreviations: NMDA, N-methyl-D-aspartate; SBDPs, spectrin breakdown products; SEM, standard error of the mean; Ctrl, control.

\section{EGb 76I suppressed the calcium influx induced by glutamate}

To assess whether the cell death is triggered by the excessive calcium influx induced by glutamate, Fluo-4 AM was used to measure the amount of intracellular calcium using flow cytometry. Calcium concentration was increased after glutamate treatment, and EGb $761(20 \mu \mathrm{g} / \mathrm{mL})$ or MK-801 $(10 \mu \mathrm{M})$ decreased calcium concentration in the glutamatetreated cells (Figure 1B). The calcium influx induced by glutamate increased the expression of calpain 2 and the amount of its specific spectrin breakdown products (SBDPs) which are cleaved by calpains. ${ }^{18}$ Similarly, our results showed that glutamate increased the expression of calpain 2 and the amount of SBDPs; however, EGb 761 or MK-801 treatment decreased the expression of calpain 2 or the amount of SBDPs remarkably (Figure 1C). Therefore, EGb 761 ameliorated the glutamate-induced excitotoxicity through the inhibition of calcium overloading.

\section{EGb 76I inhibits glutamate-mediated apoptosis}

To examine the effect of EGb 761 on its downstream mediators related to calcium accumulation, we examined the expression of caspase 3 and PARP, which play an important role in neuronal cell death. After 24 hours of exposure to $20 \mu \mathrm{g} / \mathrm{mL}$ EGb 761 or $10 \mu \mathrm{M}$ MK-801, caspase 3 decreased by $63.8 \%$ and $80.1 \%$, whereas PARP decreased by $48.7 \%$ and $62.5 \%$, respectively (Figure $2 \mathrm{~A}$ and $\mathrm{B}$ ). Then, we studied the effect of EGb 761 on Bcl-2 expression as Bcl-2 plays an important physiological role in neuronal activity, the regulation of intracellular calcium levels, and mitochondrial dynamics. ${ }^{19}$ A profound decrease was found in the expression of Bcl-2 after a treatment with glutamate, whereas MK-801 and EGb 761 pretreatment significantly reversed the decrease in Bcl-2 expression (Figure 2C). Meanwhile, the ratio of $\mathrm{Bcl}-2$ and Bax was significantly increased after MK-801 or EGb 761 administration, and the cytosolic release 
A

$\begin{array}{rllll}\text { Glu }(100 \mathrm{mM}) & - & + & + & + \\ \text { MK-801 }(10 \mu \mathrm{M}) & - & - & + & - \\ \text { EGb } 761(20 \mu \mathrm{g} / \mathrm{mL}) & - & - & - & +\end{array}$

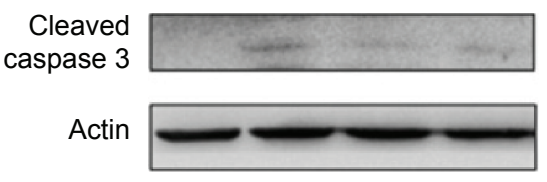

B

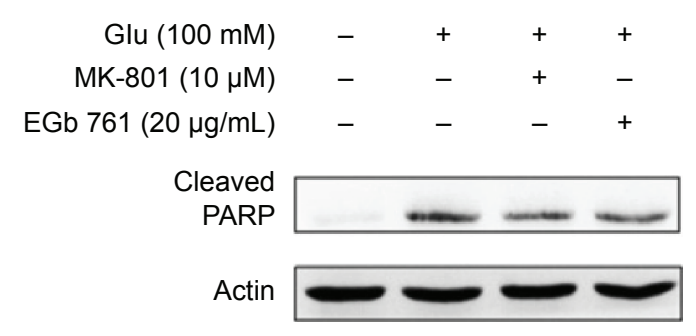

C

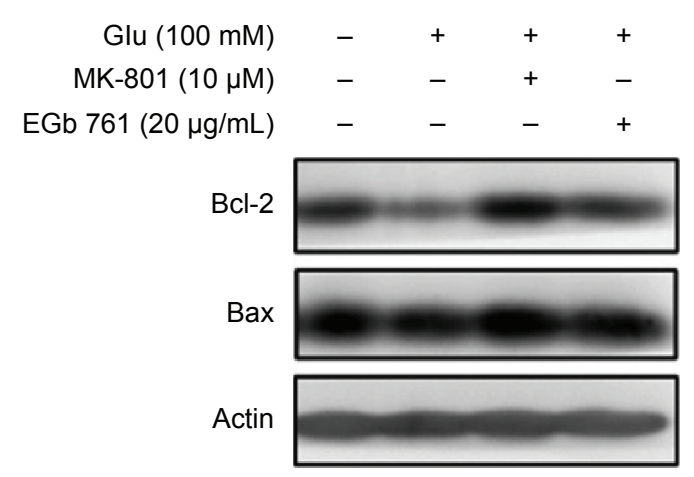

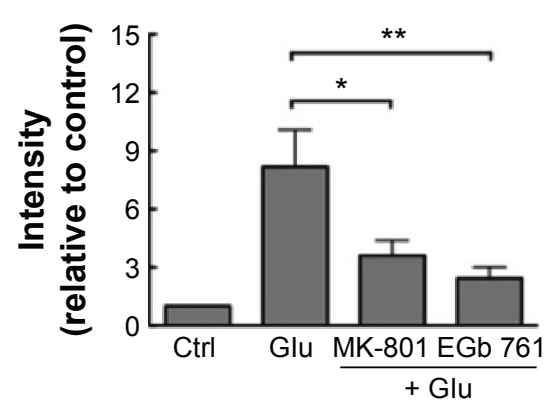

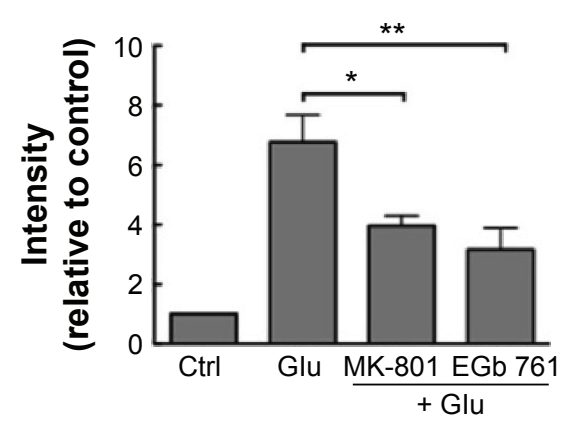

D

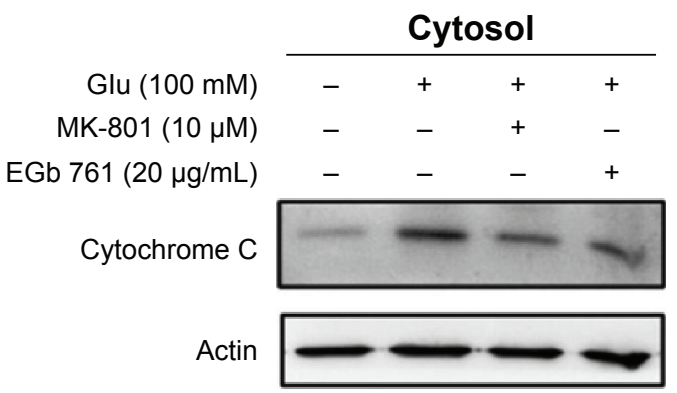

Figure 2 Treatment with EGb 76 I downregulates apoptosis markers induced by glutamate.

Notes: Western blot for cleaved caspase 3 (A), and cleaved PARP (B) after glutamate treatment of 24 hours (left panel). Relative intensities of cleaved caspase 3 and cleaved PARP are presented in the right panel (mean $\pm S E M, n=3$ ). (C) Protein expression of $B c l-2$ and Bax is detected after a treatment with glutamate for I 2 hours. The relative ratio of $\mathrm{Bcl}-2$ and $\mathrm{Bax}$ is normalized to the actin control. (D) Immunoblotting is performed on cytoplasmic extracts using an anti-cytochrome $\mathrm{C}$ antibody after a glutamate treatment for 12 hours. Actin is used as an internal control. $* P<0.05 ; * * P<0.01$.

Abbreviations: PARP, poly(adenosine diphosphate-ribose) polymerase; SEM, standard error of the mean; Ctrl, control.

of mitochondrial cytochrome $\mathrm{C}$ induced by glutamate was suppressed (Figure 2D). Therefore, the cytosolic calcium accumulation induced by glutamate triggered a caspasedependent cell death that was rescued by MK-801 or EGb 761 through the regulation of Bcl-2 expression.

\section{EGb 76I attenuates TBP/79Q aggregation and apoptosis in the TBP/79Q-EGFP cells}

To determine the neuroprotective effects of EGb 761 in the cellular model of SCA 17, the expression of TBP containing 36 or 79 glutamines fused to EGFP (TBP/36Q-EGFP and TBP/79Q-EGFP cells) was induced by doxycycline in
SH-SY5Y cells. The levels of cleaved caspase 3 and PARP were increased in the TBP/79Q-EGFP cells, but not in the TBP/36Q-EGFP-expressing cells. EGb 761 decreased the expression of cleaved caspase- 3 and PARP by $65.5 \%$ and $41.2 \%$, respectively, after the induction of TBP/79Q-EGFP cells (Figure 3A and B). Pretreatment with EGb 761 inhibited TBP aggregation by $59.9 \%$ in the TBP/79Q-EGFP cells, and no green fluorescent protein aggregation was visible in the TBP/36Q-EGFP cells (Figure 4A). Furthermore, an SDS-insoluble aggregation was detected by the dot blot filter retardation assay stained with a TBP-specific antibody; EGb 761 reduced TBP aggregation by $58.5 \%$ in the SDS-insoluble 
A

\begin{tabular}{ccccccccc} 
& \multicolumn{3}{c}{$36 Q$} & & & \multicolumn{3}{c}{$79 Q$} \\
\cline { 2 - 4 } Doxycycline & - & + & + & & - & + & + \\
$1(20 \mu \mathrm{g} / \mathrm{mL})$ & - & - & + & & - & - & +
\end{tabular}
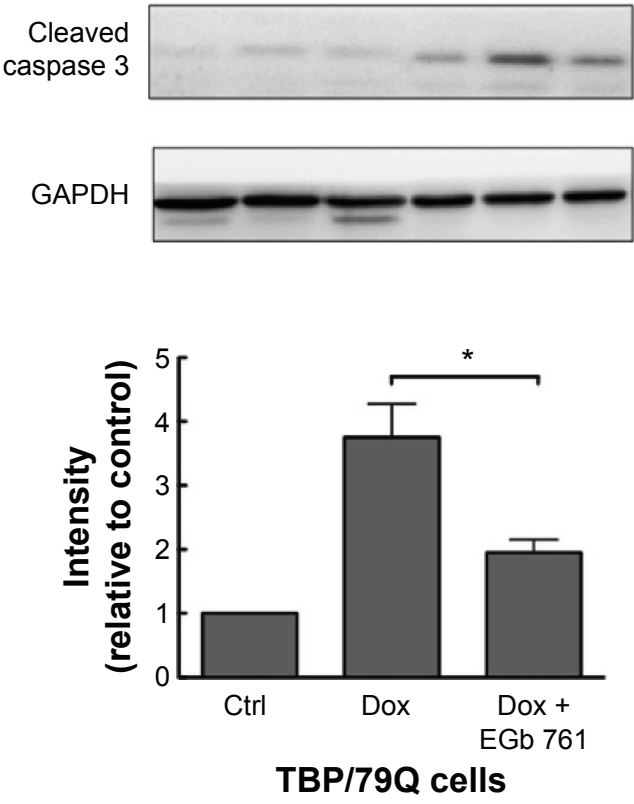

B

\begin{tabular}{lllllllll} 
& \multicolumn{3}{c}{$36 \mathbf{Q}$} & & \multicolumn{3}{c}{$79 Q$} \\
\cline { 2 - 4 } Doxycycline & - & + & + & & - & + & + \\
$1(20 \mu \mathrm{g} / \mathrm{mL})$ & - & - & + & & - & - & +
\end{tabular}

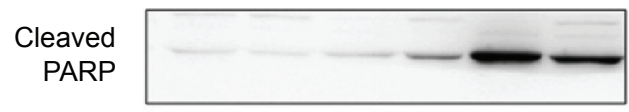

Actin
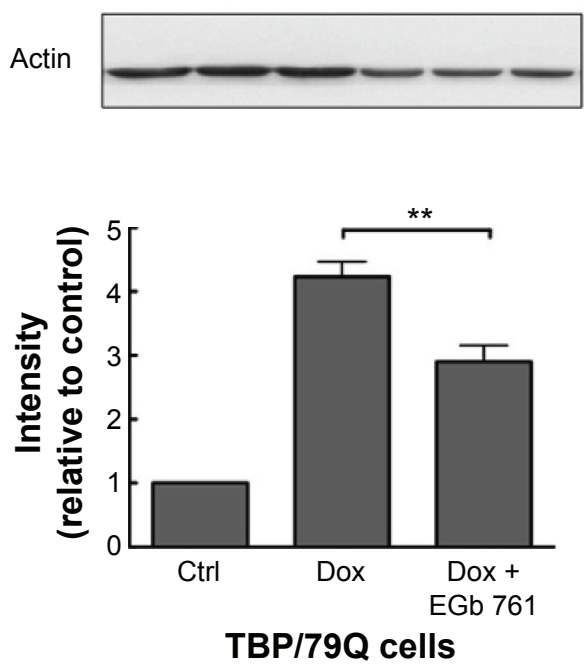

Figure 3 Inhibitory effects of EGb 76I on apoptosis in TBP/79Q-EGFP cells.

Notes: TBP expression is induced for 5 days using doxycycline, and lysates are analyzed for the expression of cleaved caspase 3 (A) and cleaved PARP (B) (top panels). Relative intensities of cleaved caspase 3 and cleaved PARP are presented in the lower panel (mean $\pm S E M, n=3$ ). $* P<0.05$; $* * P<0.01$.

Abbreviations: PARP, poly(adenosine diphosphate-ribose) polymerase; SEM, standard error of the mean; TBP, TATA box-binding protein; Ctrl, control; Dox, doxycycline; GAPDH, glyceraldehyde 3-phosphate dehydrogenase.

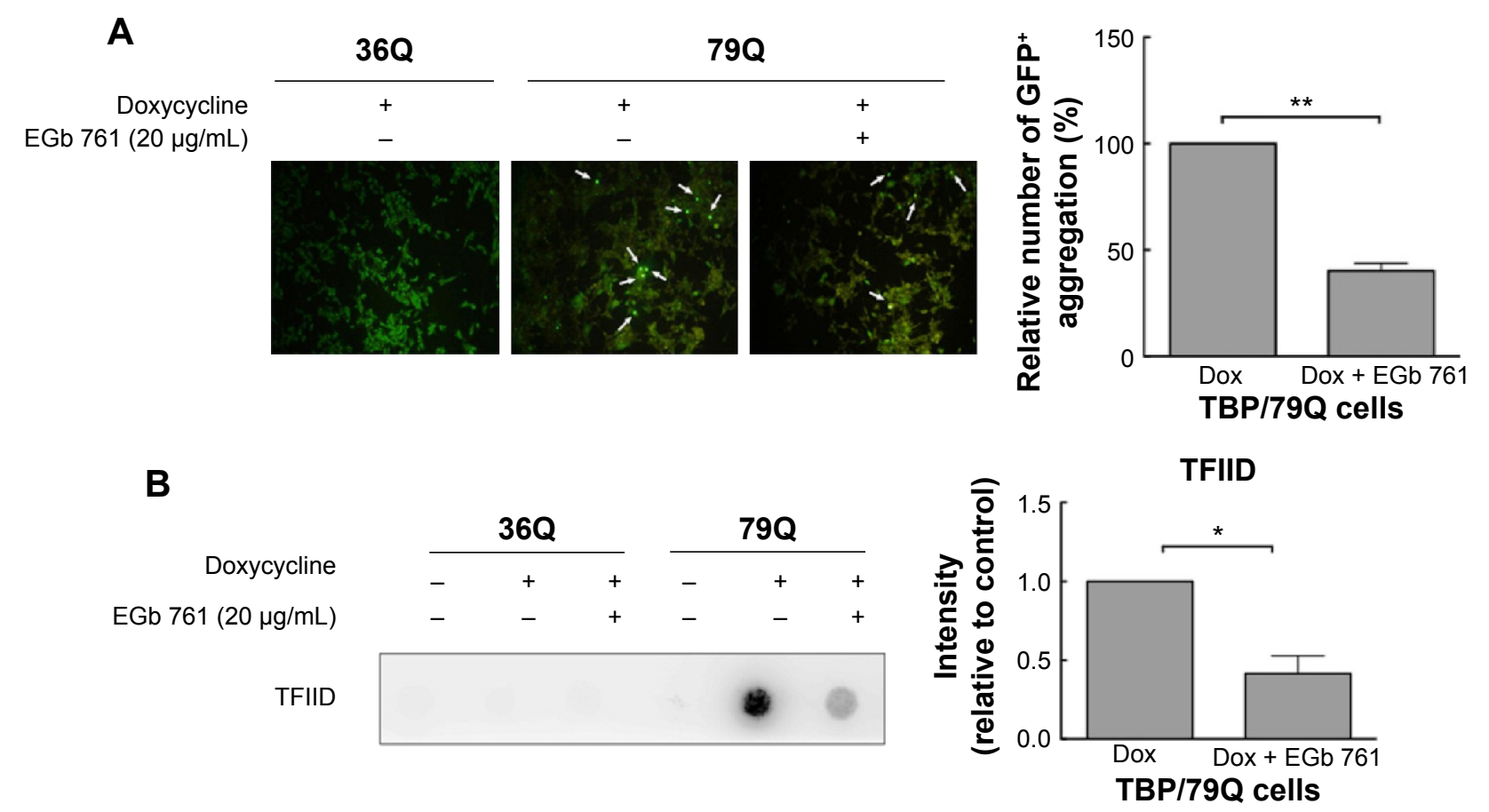

Figure 4 Suppression of protein aggregation by EGb $76 \mathrm{I}$ in the TBP/79Q-EGFP cells.

Notes: (A) Representative images of protein aggregation (arrows) in TBP-expressing cells. Cells are induced for 5 days using doxycycline to express TBP/36Q-EGFP or TBP/79Q-EGFP. (B) After TBP expression, insoluble materials are resuspended in an SDS-containing buffer. Cell lysates $(20 \mu \mathrm{g} / \mu \mathrm{L})$ are bound to a cellulose acetate membrane and are subjected to anti-TFIID dot blot immunoassay. $* P<0.05 ; * * P<0.01$.

Abbreviations: EGFP, enhanced green fluorescent protein; GFP, green fluorescent protein; SDS, sodium dodecyl sulfate; SEM, standard error of the mean; TBP, TATA box-binding protein; TFIID, transcription factor II D; Dox, doxycycline. 
materials of TBP/79Q-EGFP cells (Figure 4B). Overall, these results suggest that apoptosis is activated by $\mathrm{TBP} / 79 \mathrm{Q}$ aggregation, and $\mathrm{EGb} 761$ decreases apoptosis by inhibiting TBP/79Q aggregation.

\section{EGb 76I ameliorated the motor function of the SCA 17 transgenic mice}

Cerebellum plays an important role in motor control, and it is the brain region most affected by SCAs. In the present study, $100 \mathrm{mg} / \mathrm{kg}$ EGb 761 was administrated intraperitoneally that there was no observed effect on embryo-fetal development in mice ${ }^{20}$ and was also used for in vivo study of neurodegenerative disease. ${ }^{21,22}$ To examine whether EGb 761 improves motor function in the SCA 17 transgenic mice, we examined the highly abundant cerebellar Purkinje cells using calbindin staining at the age of 24 weeks. ${ }^{23,24}$ The Purkinje cells of the wild-type mice formed a compact layer, but the alignment of Purkinje cells was disrupted in the SCA 17 transgenic mice. The disrupted stratification was restored by the EGb 761 treatment in the SCA 17 transgenic mice (Figure 5A). Furthermore, EGb 761 inhibited TBP aggregation in the SCA 17 transgenic mice (Figure 5B). The impairment of motor functions in the SCA 17 transgenic mice was observed at the age of 11 weeks as demonstrated by the rotarod experiments that analyze motor coordination (Figure 5C). For 20 weeks, $100 \mathrm{mg} / \mathrm{kg}$ EGb 761 was administered intraperitoneally every other day to the SCA 17 transgenic mice from the age of 4 weeks. We observed that the EGb 761-treated SCA 17 transgenic mice showed increased rotarod performance by $64.8 \%$ and $54.7 \%$ compared with the untreated SCA 17 transgenic mice at the age of 15 weeks and 17 weeks, respectively (Figure 5C).
To determine whether calcium homeostasis is disrupted in the SCA 17 transgenic mice, the cerebellar extracts were examined for SBDPs generated by calpains. The amount of calpain-specific SBDPs was increased in the cerebellum of the SCA 17 transgenic mice, but it was decreased in the EGb 761-treated SCA 17 transgenic animals (Figure 5D). To conclude, we observed the impairment of motor function and the activation of intracellular calcium signaling in the cerebellum of the SCA 17 transgenic mice that were attenuated by EGb 761, suggesting that EGb 761 ameliorates motor dysfunction by inhibiting the abnormal calcium signaling and TBP aggregation in the SCA 17 mice.

\section{Discussion}

In the present study, we examined the molecular mechanisms of EGb 761 treatment in decreasing cell death caused by excitotoxicity. Our results show that EGb 761 inhibits the glutamate-induced apoptosis in SH-SY5Y cells through the suppression of excessive calcium influx and subsequent inhibition of excitotoxicity and cell death demonstrated by the decreased expression of cleaved caspase 3 and PARP. Excessive influx of calcium is implied in neurodegenerative diseases and results in excitotoxicity and subsequent neuronal injury. Furthermore, EGb 761 was protective against cell death induced by calcium overloading in vitro (Figures 1 and 2) and in vivo. EGb 761 decreased the amount of calpain-specific SBDPs in the cerebellum of the SCA 17 transgenic mice (Figure 5B). This observation suggests that EGb 761 may decrease calcium overloading in SCA 17 and rescue neuronal cells from injury.

Patients with SCA 17 develop a variety of neuroanatomical abnormalities, including the formation of neuronal inclusions
A

Calbindin

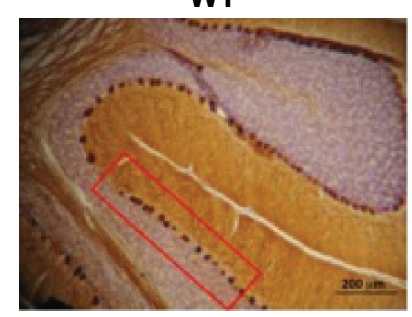

B

1TBP18
TG

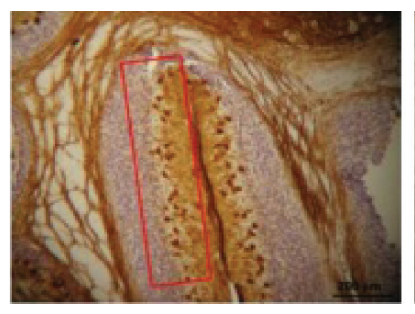

TG

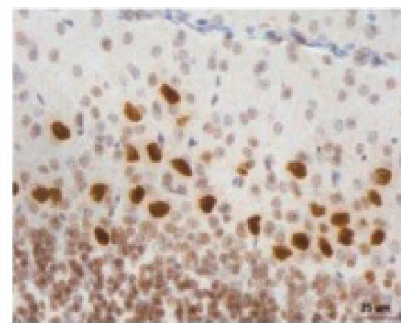

TG + EGb 761

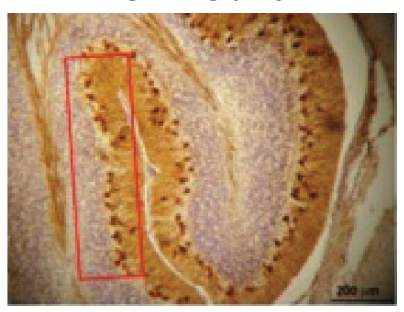

$\mathrm{TG}+\mathrm{EGb} 761$

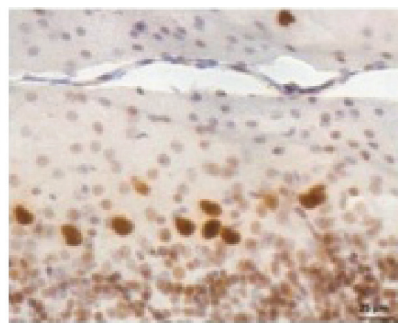

Figure 5 (Continued) 
C

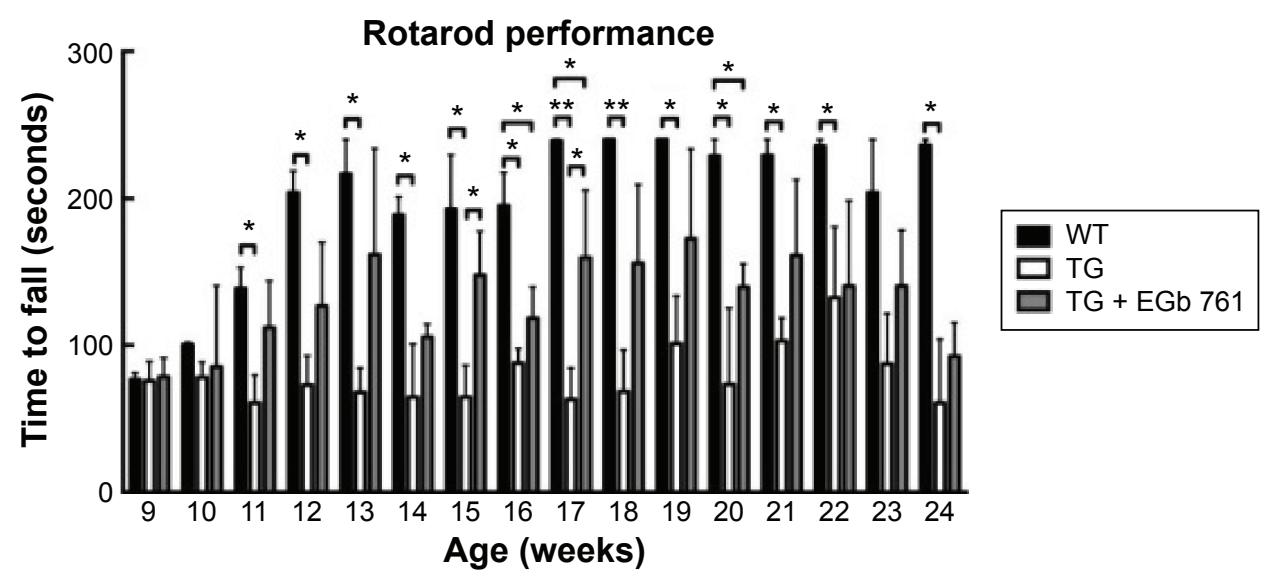

D
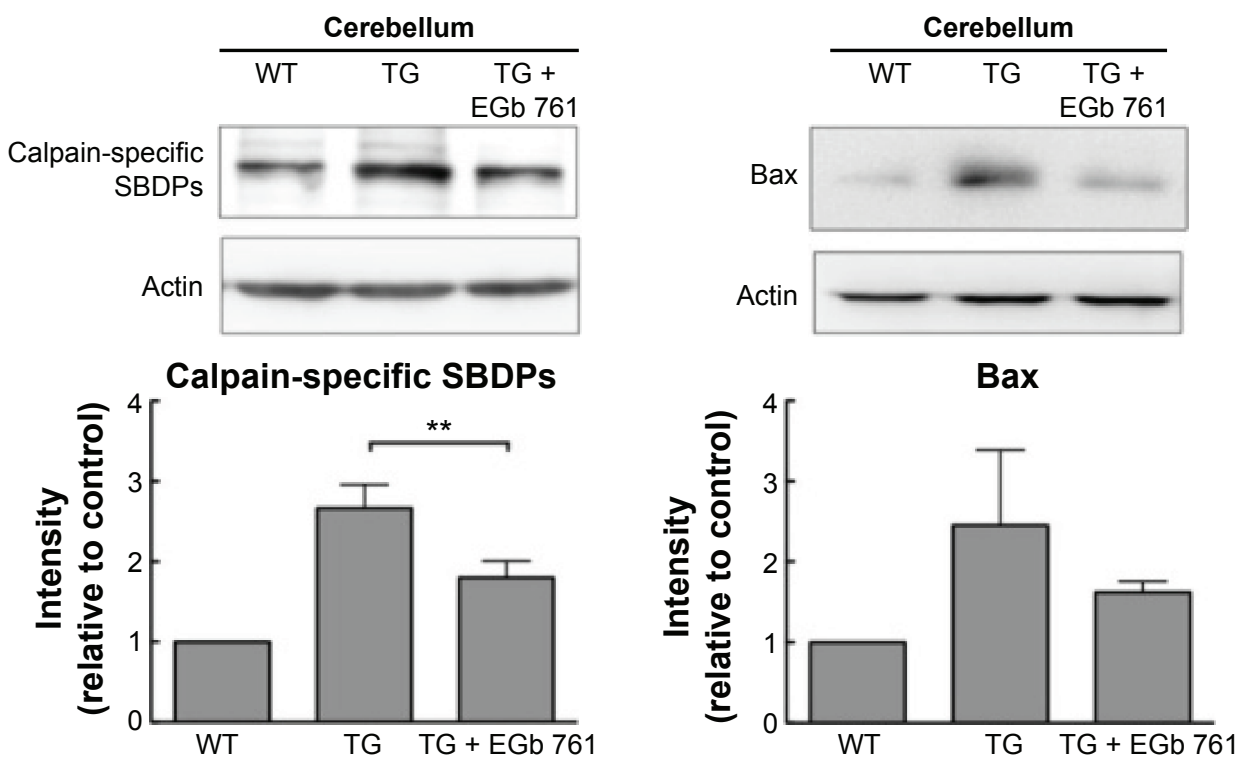

Figure 5 Protective effects of EGb 761 in the SCA 17 transgenic mice (TG).

Notes: (A) Calbindin immunostaining reaction developed with DAB in the cerebellar vermis of WT and SCA I7 transgenic mice. (B) IHC staining with ITBPI8 in vermis of WT (arrows) and SCA 17 transgenic mice. The images in (B) represent the area shown by the red rectangles in (A). (C) The latency to fall is recorded every week (9-24 weeks) in the accelerating rotarod test. (D) The cerebellar extracts of mice treated in vivo are immunostained against SBDPs (left panel). Relative intensity of calpainspecific SBDPs is presented in the right panel (mean \pm SEM, $n=3$ ). $* p<0.05 ; * * P<0.0$ I.

Abbreviations: DAB, 3,3'-diaminobenzidine; IHC, immunohistochemistry; SBDPs, spectrin breakdown products; SCA I7, spinocerebellar ataxia type I7; SEM, standard error of the mean; TBP, TATA box-binding protein; WT, wild type.

and neuronal loss affecting particularly the Purkinje cells of the cerebellum, and subsequent cerebellar atrophy. A previous study also indicated that quality control may be inefficient for polyQ proteins, and one of the therapeutic strategies suggested is to assist the correct folding of polyQ proteins to reduce aggregation. ${ }^{2,25,26}$ The aggregation of misfolded proteins has been documented in polyQ diseases such as Huntington's disease, Alzheimer's disease, and SCA 3. The present study demonstrates that EGb 761 inhibits the SDS-insoluble TBP aggregation in the TBP/79Q cells (Figures 3 and 4). These data suggest that EGb 761 alleviates cell toxicity by inhibiting the TBP/79Q aggregation.

The rotarod performance is used to evaluate motor coordination in animal models. In this study, motor coordination declined in the SCA 17 transgenic mice compared with the wild-type mice, and the intraperitoneal administration of EGb 761 improved the rotarod performance of the SCA 17 transgenic mice at the age of 15 weeks and 17 weeks (Figure 5C), but rotarod performance was not significantly increased from the age of 18 weeks to 24 weeks. These data suggest that the motor dysfunction is delayed by the administration of EGb 761 in the SCA 17 transgenic mice.

\section{Conclusion}

In conclusion, in vitro application of EGb 761 alleviates excitotoxicity and the subsequent apoptosis by inhibiting the apoptotic pathways induced by the aggregation of TBP/79Q. Moreover, in vivo EGb 761 delays the motor deficit and 
neuropathological alterations found in the SCA 17 transgenic mice. These data suggest that EGb 761 may be a potential therapeutic agent for SCA 17.

\section{Acknowledgments}

This study was supported by the grants NSC 100-2325B-002-064, NSC 101-2325-B-002-057, NSC 102-2325B-002-055, MOST 103-2325-B-003-002, and MOST 104-2325-B-003-002 and from the Ministry of Science and Technology, and 103T3040B00, 104T3040C0, 104T3040D0, and 105T3040D0 from the National Taiwan Normal University, Taipei, Taiwan, Republic of China.

\section{Disclosure}

The authors report no conflicts of interest in this work.

\section{References}

1. Fujigasaki H, Martin JJ, DeDeyn PP, et al. CAG repeat expansion in the TATA box-binding protein gene causes autosomal dominant cerebellar ataxia. Brain. 2001;124:1939-1947.

2. Nakamura K, Jeong SY, Uchihara T, et al. SCA 17, a novel autosomal dominant cerebellar ataxia caused by an expanded polyglutamine in TATA-binding protein. Hum Mol Genet. 2001;10(14):1441-1448.

3. Zühlke C, Hellenbroich Y, Dalski A, et al. Different types of repeat expansion in the TATA-binding protein gene are associated with a new form of inherited ataxia. Eur J Hum Genet. 2001;9(3):160-164.

4. Koch P, Breuer P, Peitz M, et al. Excitation-induced ataxin-3 aggregation in neurons from patients with Machado-Joseph disease. Nature. 2011;480(7378):543-546.

5. Hübener J, Weber JJ, Richter C, et al. Calpain-mediated ataxin-3 cleavage in the molecular pathogenesis of spinocerebellar ataxia type 3 (SCA3). Hum Mol Genet. 2013;22(3):508-518.

6. Simoes AT, Goncalves N, Koeppen A, et al. Calpastatin-mediated inhibition of calpains in the mouse brain prevents mutant ataxin 3 proteolysis, nuclear localization and aggregation, relieving MachadoJoseph disease. Brain. 2012;135(pt 8):2428-2439.

7. Tang TS, Tu H, Chan EY, et al. Huntingtin and huntingtin-associated protein 1 influence neuronal calcium signaling mediated by inositol$(1,4,5)$ triphosphate receptor type 1. Neuron. 2003;39(2):227-239.

8. Tang TS, Slow E, Lupu V, et al. Disturbed Ca2+ signaling and apoptosis of medium spiny neurons in Huntington's disease. Proc Natl Acad Sci U S A. 2005;102(7):2602-2607.

9. Ramassamy C. Emerging role of polyphenolic compounds in the treatment of neurodegenerative diseases: a review of their intracellular targets. Eur J Pharmacol. 2006;545(1):51-64.

10. Chandrasekaran K, Mehrabian Z, Spinnewyn B, Drieu K, Fiskum G. Neuroprotective effects of bilobalide, a component of the Ginkgo biloba extract (EGb 761), in gerbil global brain ischemia. Brain Res. 2001;922(2):282-292.
11. Oyama Y, Chikahisa L, Ueha T, Kanemaru K, Noda K. Ginkgo biloba extract protects brain neurons against oxidative stress induced by hydrogen peroxide. Brain Res. 1996;712(2):349-352.

12. Bastianetto S, Ramassamy C, Doré S, Christen Y, Poirier J, Quirion R. The Ginkgo biloba extract (EGb 761) protects hippocampal neurons against cell death induced by beta-amyloid. Eur J Neurosci. 2000; 12(6):1882-1890.

13. Diamond BJ, Shiflett SC, Feiwel N, et al. Ginkgo biloba extract: mechanisms and clinical indications. Arch Phys Med Rehabil. 2000; 81(5):668-678.

14. Kung PJ, Tao YC, Hsu HC, et al. Indole and synthetic derivative activate chaperone expression to reduce polyQ aggregation in SCA 17 neuronal cell and slice culture models. Drug Des Devel Ther. 2014; 8:1929-1939.

15. Chang YC, Lin CY, Hsu CM, et al. Neuroprotective effects of granulocyte-colony stimulating factor in a novel transgenic mouse model of SCA 17. J Neurochem. 2011;118(2):288-303.

16. Rothman SM, Olney JW. Excitotoxicity and the NMDA receptor - still lethal after eight years. Trends Neurosci. 1995;18(2):57-58.

17. Sun ZW, Zhang L, Zhu SJ, Chen WC, Mei B. Excitotoxicity effects of glutamate on human neuroblastoma SH-SY5Y cells via oxidative damage. Neurosci Bull. 2010;26(1):8-16.

18. Miao Y, Dong LD, Chen J, Hu XC, Yang XL, Wang Z. Involvement of calpain/p35-p25/Cdk5/NMDAR signaling pathway in glutamateinduced neurotoxicity in cultured rat retinal neurons. PLoS One. 2012;7(8):e42318.

19. Hardwick JM, Soane L. Multiple functions of BCL-2 family proteins. Cold Spring Harb Perspect Biol. 2013;5(2):a008722.

20. Koch E, Nöldner M, Leuschner J. Reproductive and developmental toxicity of the Ginkgo biloba special extract EGb 761 in mice. Phytomedicine. 2013;12(1):90-97.

21. Mdzinarishvili A, Sumbria R, Lang D, Klein J. Ginkgo extract EGb 761 confers neuroprotection by reduction of glutamate release in ischemic brain. J Pharm Pharm Sci. 2012;15(1):94-102.

22. Rojas P, Serrano-García N, Mares-Sámano JJ, Medina-Campos ON, Pedraza-Chaverri J, Ogren SO. EGb761 protects against nigrostriatal dopaminergic neurotoxicity in 1-methyl-4-phenyl-1,2,3,6-tetrahydropyridine-induced Parkinsonism in mice: role of oxidative stress. Eur J Neurosci. 2008;28(1):41-50.

23. Barski JJ, Hartmann J, Rose CR, et al. Calbindin in cerebellar Purkinje cells is a critical determinant of the precision of motor coordination. J Neurosci. 2003;23(8):3469-3477.

24. Whitney ER, Kemper TL, Rosene DL, Bauman ML, Blatt GJ. Calbindin-D28k is a more reliable marker of human Purkinje cells than standard Nissl stains: a stereological experiment. J Neurosci Methods. 2008;168(1):42-47.

25. Park SH, Kukushkin Y, Gupta R, et al. PolyQ proteins interfere with nuclear degradation of cytosolic proteins by sequestering the Sislp chaperone. Cell. 2013;154(1):134-145.

26. Ross C, Poirier M. Protein aggregation and neurodegenerative disease. Nat Med. 2004;10(suppl):10-17.

\section{Publish your work in this journal}

Drug Design, Development and Therapy is an international, peerreviewed open-access journal that spans the spectrum of drug design and development through to clinical applications. Clinical outcomes, patient safety, and programs for the development and effective, safe, and sustained use of medicines are a feature of the journal, which

\section{Dovepress}

has also been accepted for indexing on PubMed Central. The manuscript management system is completely online and includes a very quick and fair peer-review system, which is all easy to use. Visit http://www.dovepress.com/testimonials.php to read real quotes from published authors. 